\title{
Influence of remote monitoring on long-term cardiovascular outcomes after cardioverter-defibrillator implantation
}

\author{
Guilherme Portugal ${ }^{*, 1}$, Pedro Cunha ${ }^{1}$, Bruno Valente ${ }^{1}$, Joana Feliciano ${ }^{1}$, Ana Lousinha ${ }^{1}$, Sandra Alves ${ }^{1}$, \\ Manuel Braz ${ }^{1}$, Ricardo Pimenta ${ }^{1}$, Ana Sofia Delgado ${ }^{1}$, Mário Oliveira ${ }^{1}$, Rui C. Ferreira ${ }^{1}$ \\ Department of Cardiology, Hospital de Santa Marta, Centro Hospitalar Lisboa Central EPE, Lisbon, Rua de Santa Marta 50, 1169-024 Lisboa, Portugal
}

\section{A R T I C L E I N F O}

\section{Article history:}

Received 22 March 2016

Accepted 26 July 2016

Available online 27 July 2016

\section{Keywords:}

Remote monitoring

ICD

\begin{abstract}
A B S T R A C T
Aims: Device-based remote monitoring (RM) has been linked to improved clinical outcomes at short to mediumterm follow-up. Whether this benefit extends to long-term follow-up is unknown. We sought to assess the effect of device-based RM on long-term clinical outcomes in recipients of implantable cardioverter-defibrillators (ICD). Methods: We performed a retrospective cohort study of consecutive patients who underwent ICD implantation for primary prevention. RM was initiated with patient consent according to availability of RM hardware at implantation. Patients with concomitant cardiac resynchronization therapy were excluded. Data on hospitalizations, mortality and cause of death were systematically assessed using a nationwide healthcare platform. A Cox proportional hazards model was employed to estimate the effect of RM on mortality and a composite endpoint of cardiovascular mortality and hospital admission due to heart failure (HF).

Results: 312 patients were included with a median follow-up of 37.7 months (range 1 to 146). 121 patients (38.2\%) were under RM since the first outpatient visit post-ICD and 191 were in conventional follow-up. No differences were found regarding age, left ventricular ejection fraction, heart failure etiology or NYHA class at implantation. Patients under RM had higher long-term survival (hazard ratio [HR] 0.50, CI 0.27-0.93, p = 0.029) and lower incidence of the composite outcome (HR 0.47, CI 0.27-0.82, p = 0.008). After multivariate survival analysis, overall survival was independently associated with younger age, higher LVEF, NYHA class lower than 3 and RM.

Conclusion: RM was independently associated with increased long-term survival and a lower incidence of a composite endpoint of hospitalization for HF or cardiovascular mortality.
\end{abstract}

(c) 2016 Elsevier Ireland Ltd. All rights reserved.

\section{Introduction}

Technological developments and expanded indications have translated into a large population of recipients of implantable electronic cardiac devices. There has been a significant increase in the number of patients submitted to implantation of cardioverter-defibrillators (ICD). This growing population represents a unique challenge regarding their follow-up, requiring an experienced team with in-depth knowledge of device programming and potential complications [1]. To this date, optimal clinical resource allocation regarding adequate follow-up to these patients is not yet established.

In this setting, remote monitoring (RM) is poised as an optimal tool in the intensive and continuous follow-up of ICD patients. Published data supports the safety and efficacy of this intervention [1]. However,

\footnotetext{
* Corresponding author at: Department of Cardiology, Hospital de Santa Marta, Rua de Santa Marta 50, 1169-024 Lisboa, Portugal.

E-mail address: Gfportugal@gmail.com (G. Portugal).

1 This author takes responsibility for all aspects of the reliability and freedom from bias of the data presented and their discussed interpretation
}

data regarding potential clinical benefits has been scarce, with a short duration of follow-up and mainly limited to specific ICD brands.

In this study we aimed to assess the long-term clinical benefits of RM in a population of patients with ICD for primary prevention of sudden cardiac death. As such, we sought to investigate the effect of RM on hospital admissions for heart failure (HF), cardiovascular death and all-cause death.

\section{Methods}

We performed a retrospective cohort study in patients who underwent ICD implantation for primary prevention and compared a group followed with use of RM (RM +) versus a group on conventional outpatient clinic follow-up ( $R M-$ ). Consecutive patients referred to a tertiary center for implantation of an ICD between December 2002 and October 2014 were included. The study population was divided in two groups, according to the modality of follow-up: RM + versus RM - . The protocol was approved by the Ethics Committee of our institution.

Implantation of an ICD was performed according to international guidelines [2] regarding patients with systolic dysfunction or primary channelopathies. ICD implantation in the setting of hypertrophic cardiomyopathy was performed according to disease-specific guidelines [3]. Patients with indication for implantation for secondary prevention or with concurrent implantation of cardiac resynchronization 
therapy (CRT) were excluded. ICD patients who underwent an upgrade to a CRT system during follow-up were censored for analysis after the procedure.

RM was offered to patients whenever available as offered by the device company with patient informed consent. The equipment was provided free of charge at the first outpatient visit after ICD implantation. Patients were then scheduled for a hospital visit every 12 months, with transmissions performed every 3 months, during the study period. Reports were reviewed by trained technicians who would alert the attending physician in case of relevant events. Additionally, RM alerts were transmitted in response to abnormal events such as delivered shocks, detection of atrial fibrillation episodes or abnormal values of the various ICD parameters. In this case, patients were summoned in the following $48 \mathrm{~h}$ for an in-person consultation with an electrophysiologist in order to review of the episode.

Conventional follow-up consisted of a patient visit 1 month after implantation and every 4 months thereafter. Device interrogation was performed in all visits, and relevant events (e.g. lead impedance or other parameters changes, atrial fibrillation detection or appropriate/inappropriate therapy) were registered. The patient was then observed by an electrophysiologist, and changes in device programming, reintervention, drug therapy or indication for ablation were employed as deemed appropriate.

Data collection regarding arrhythmic events, device programming and appropriate and inappropriate therapy via ICD was prospectively inputted into a database consisting of all patients. These records were then assessed at the time of this study for possible missing data and completed whenever possible. Patients were considered lost to follow-up when the last outpatient visit was $>18$ months with no further scheduled visits.

Information on hospitalizations and mortality was assessed with the use of a nationwide healthcare platform ("Plataforma de Dados de Saúde") integrating records of 9 out of 10 of the referring hospitals and the National Registry on Mortality. Patient records were systematically reviewed, and information was collected regarding hospital admissions and all-cause mortality. The cause of hospitalization was determined according to an International Classification of Disease-9 (ICD-9) based system as coded by the discharging hospital. Likewise, cause of mortality was determined according to the same ICD-9 based codification. The physician responsible for assessing study outcomes was blinded in regard to the modality of follow-up (remote monitoring vs in-office)

The main measured endpoint was time to all-cause death. A secondary endpoint consisted of a composite endpoint of time to hospitalization for $\mathrm{HF}$ or cardiovascular death. In addition, we determined independent predictors of time to all-cause death with use of a stepwise approach.

\section{Statistical analysis}

Continuous variables were displayed as mean and standard deviation where they followed a normal distribution, and in terms of their median and interquartile range where they did not. Qualitative variables were expressed in terms of frequency and percentage. Baseline comparisons were performed using the chi-square test for qualitative data and the t-student test for continuous variables.

The analysis of main outcome of time to admission for HF and mortality was performed by Kaplan-Meier curves, log-rank test for comparisons and use of the Cox proportional hazards model for calculation of the hazard ratio (HR). A stepwise approach was employed to determine independent predictors of all-cause death. Initially, univariate predictors of all-cause death were determined with use of a Cox regression model. Variables with a significance level $<0.1$ were subsequently included in the multivariate survival model. We additionally performed subgroup analysis for age, left ventricular ejection fraction, etiology and device brand. A two-sided P-value $<0.05$ was considered statistically significant. All statistical analysis was performed with the software package STATA 12 (Statacorp).

\section{Results}

\subsection{Study population}

A total of 923 patients received an ICD during this period. Of these, 611 patients were excluded on the basis of concomitant CRT (473 patients) or implantation in the setting of secondary prevention (138 patients). After exclusion, a total of 312 patients underwent further analysis.

Patient characteristics at implantation are described in Table 1. Most patients had an indication for ICD in primary prevention in the setting of left ventricular systolic dysfunction (85.6\%). The etiology was ischemic cardiomyopathy in 58.5\%, idiopathic dilated cardiomyopathy in $27.1 \%$, hypertrophic cardiomyopathy in $8.1 \%$, Brugada syndrome in $3.3 \%$ and other etiologies in 3.0\% (4 patients with hypertensive heart disease, 2
Table 1

Baseline characteristics.

\begin{tabular}{llll}
\hline & $\begin{array}{l}\text { Remote } \\
\text { monitoring }\end{array}$ & Standard care & $\mathrm{P}=$ \\
\hline Number & $121(38.2 \%)$ & $191(61.8 \%)$ & \\
Age at implantation (years) & $56.9+/-1.3$ & $57.9+/-1.1$ & $\mathrm{NS}$ \\
Male sex & $104(85.9 \%)$ & $170(89.0 \%)$ & $\mathrm{NS}$ \\
Left ventricular ejection fraction (\%) & $30.0+/-1.14$ & $28.2+/-0.69$ & $\mathrm{NS}$ \\
Etiology & & & $\mathrm{NS}$ \\
- Ischaemic & $66(54.5 \%)$ & $117(61.2 \%)$ & \\
- Dilated cardiomyopathy & $35(28.9 \%)$ & $51(26.7 \%)$ & \\
- Hypertrophic cardiomyopathy & $11(9.1 \%)$ & $14(7.3 \%)$ & \\
- Brugada syndrome & $4(3.3 \%)$ & $6(3.1 \%)$ & \\
- Other & $6(4.9 \%)$ & $3(1.5 \%)$ & \\
NYHA class & & & \\
I & $24(19.8 \%)$ & $31(16.2 \%)$ & \\
II & $79(65.3 \%)$ & $133(69.6 \%)$ & \\
III & $17(14.0 \%)$ & $26(13.6 \%)$ & \\
IV & $1(0.8 \%)$ & $1(0.5 \%)$ & \\
Device Brand & & & \\
- Biotronik & $58(78.4 \%)$ & $16(21.6 \%)$ & \\
- Boston Scientific & $25(31.6 \%)$ & $54(68.3 \%)$ & \\
- Medtronic & $25(34.7 \%)$ & $47(65.3 \%)$ & \\
- Sorin & $11(19.3 \%)$ & $46(80.7 \%)$ & \\
- St- Jude Medical & $2(5.9 \%)$ & $32(94.1 \%)$ & \\
\hline
\end{tabular}

patients with congenital heart disease, 2 patients with valvular heart disease and 1 patients with left ventricle non-compacted cardiomyopathy).

After stratification for RM, 121 patients were under remote monitoring $(\mathrm{RM}+, 38.2 \%)$ and 191 on conventional follow-up (RM-, 61.8\%).

The mean ejection fraction was $28.9 \%$, with no difference between groups regarding left ventricular systolic function (LVEF, 30.0\% +/1.14 vs. $28.2+/-0.69 \%, p=N S)$. No significant differences were found regarding age at implantation (RM vs. controls; $56.9+/-1.3$ vs. $57.9+/-1.1$ yrs., $p=N S$ ).

\subsection{Follow-up}

Median follow-up was 37.7 months (IQR 18.8-65.8), with 4 patients (1.28\%) lost to follow-up during the 12 year study period ( 1 in the $\mathrm{RM}+$ group and 3 in the RM - group, $\mathrm{p}=\mathrm{NS}$ ). Mean follow-up duration was higher in patients under RM (48.4 months +/2.7 vs $40.3+/-2.2, \mathrm{p}=0.021)$. Data on overall mortality was available for the remaining 307 patients, and was used to calculate the Kaplan-Meier survival estimates (Fig. 1).

\subsection{Primary outcome}

Regarding overall mortality, RM was significantly associated with increased survival with a hazard ratio of 0.51 (Confidence interval $0.24-0.84, \mathrm{p}=0.021$ ). This difference, manifested early during followup and increased throughout the study time, suggests a cumulative benefit of RM with length of follow-up.

In addition, we performed a stepwise multivariate analysis for determination of independent predictors of survival at follow-up, which showed that lower age, higher LVEF, NYHA functional class $<3$ and RM were independent predictors of survival (Table 2).

To test whether increased availability of RM on recent years could explain the improved outcomes in these patients, we controlled for the effect of year of implantation by patients into quartiles according to year of implantation. We found no effect of implantation year on overall survival (Cox regression, HR 0.99, CI 0.99-1.00, $\mathrm{p}=0.557$ ) nor was the effect of RM on mortality influenced by year of device implantation ( $\mathrm{p}$ for interaction $=0.744$ ).

The results for the subgroup analysis are presented in Fig. 2. No statistically significant differences were found regarding the effect of RM on mortality when stratifying by LVEF, age, etiology or device 
Overall mortality after stratification for remote monitoring

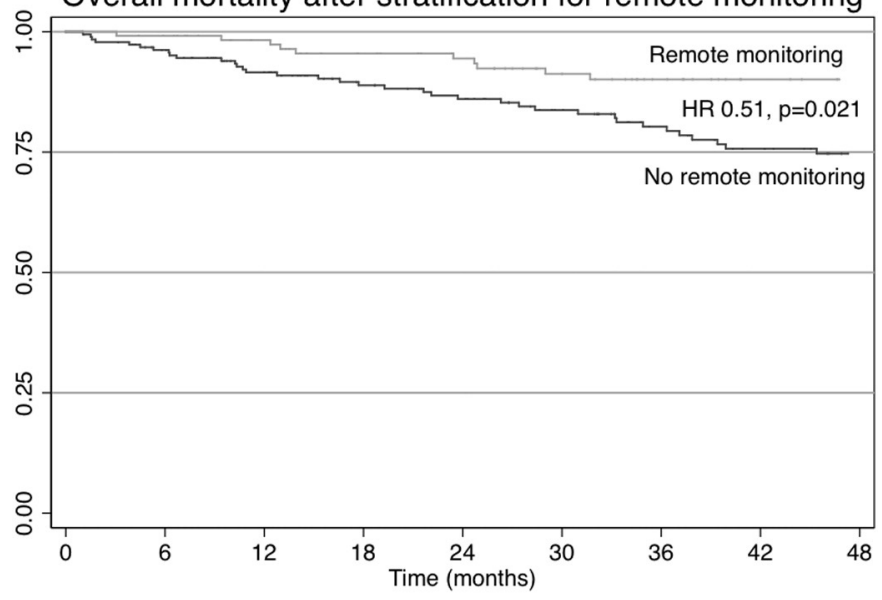

Fig. 1. Kaplan-Meier plot of overall mortality after stratification for remote monitoring. Of note, a survival benefit is noted early on and is consistent throughout the duration of follow-up.

brand ( $\mathrm{p}=\mathrm{NS}$ for all). However, a non-significant trend was observed suggesting a greater benefit of RM in patients aged older than 60 years, with $\mathrm{LVEF}<30 \%$ and with an ischemic etiology.

\subsection{Secondary outcome}

There were a total of 121 events during this period, consisting of 63 admissions for HF and 58 deaths, of which 30 were deemed to be of cardiovascular causes. These 93 events (63 admissions due to HF and 30 cardiovascular deaths) corresponded to the secondary outcome, which was observed in 17 patients in the RM group and in 48 patients in the conventional follow-up group $(\mathrm{p}=0.021)$. Kaplan-Meier plots for the composite outcome of cardiovascular death or hospitalization for HF are plotted on Fig. 3. RM was associated with a hazard ratio of 0.47 for this composite outcome ( $\mathrm{Cl} 0.27-0.82, \mathrm{p}=0.008$ ).

\section{Discussion}

The main finding of our study was a significantly lower incidence of unplanned hospital admissions for HF and overall mortality at very-long follow-up in patients under RM. This reduction in mortality was mainly driven by a reduction in cardiovascular mortality.

To further validate our findings and adjust for the potential confounding effect of other baseline characteristics, we used a stepwise approach to calculate a multivariate Cox regression model performed a multivariate regression analysis, which consistently showed the

Table 2

Survival analysis.

\begin{tabular}{|c|c|c|c|c|}
\hline & Univariate analysis & $\mathrm{p}=$ & Multivariate model & $\mathrm{p}=$ \\
\hline Age & $\begin{array}{l}\text { HR } 1.06 \\
\text { CI } 1.03-1.09\end{array}$ & $<0.001$ & HR 1.07 CI $1.04-1.10$ & $<0.001$ \\
\hline Sex (male) & $\begin{array}{l}\text { HR } 0.94 \\
\text { CI 0.44-1.99 }\end{array}$ & 0.870 & & \\
\hline $\begin{array}{l}\text { Left ventricle } \\
\text { ejection fraction }\end{array}$ & $\begin{array}{l}\text { HR } 0.95 \\
\text { CI } 0.91-0.98\end{array}$ & 0.003 & HR 0.95 CI 0.91-0.99 & 0.028 \\
\hline NYHA class $>$ II & HR 2.16 CI 1.11-4.19 & 0.023 & HR 2.52 CI 1.27-4.99 & 0.008 \\
\hline Remote monitoring & HR 0.51 CI $0.28-0.90$ & 0.021 & HR 0.50 CI $0.27-0.93$ & 0.029 \\
\hline $\begin{array}{l}\text { Device brand } \\
\text { Biotronik } \\
\text { Boston Scientific } \\
\text { Medtronic } \\
\text { Sorin } \\
\text { St- Jude Medical } §\end{array}$ & $\begin{array}{l}\text { HR } 0.94 \text { CI } 0.50-1.75 \\
\text { HR } 1.10 \text { CI } 0.61-1.97 \\
\text { HR } 0.71 \text { CI } 0.36-1.43 \\
\text { HR } 1.67 \text { CI } 0.89-3.12\end{array}$ & $\begin{array}{l}0.842 \\
0.33 \\
0.347 \\
0.109\end{array}$ & & \\
\hline
\end{tabular}

$\S$ Statistical analysis not performed owing to low number of patients on remote monitoring. protective effect of RM on mortality. As such, we determined that in our cohort, RM was an independent predictor of lower mortality at follow-up.

The benefit of RM was consistent across all tested device brands. We also did not detect a significant difference on the benefit of RM by severity of LV dysfunction, age or etiology. Although this study was not adequately powered to detect differences between subgroups, we did find a non-significant trend toward a larger reduction in mortality in higher risk patients (LVEF $<30 \%$, age over 60 years, ischemic etiology), suggesting that these patients may derive the most benefit from this technology.

Our study cohort consisted of unselected, all-comers with indication for ICD implantation for primary prevention of sudden cardiac death. As previously stated, there was no formal randomization of patients, with RM instituted according to an independent external factor, in this case the availability of the remote monitoring hardware as offered by the device company. This in effect led to a quasi-randomized patient allocation, with well balanced baseline patient characteristics and no significant differences between groups.

Data was available on hospital admissions or mortality for $98.72 \%$ of our cohort, and cause of death or hospitalization was assessed as previously mentioned in the Methods section. Patients were followed for up to 12 years with a consistent benefit of RM across the duration of follow-up.

Contemporary wireless RM systems forgo the need for manual wanded transmissions and perform automatic transmissions requiring minimal patient input. Notwithstanding, recognized gains in resource efficiency, and some clinical benefits have also been demonstrated in clinical trials. These included a greater adherence to follow-up and a faster time to physician evaluation after event occurrence [4], and also lower incidence of atrial fibrillation and stroke related hospital admissions [5], and reduced length of hospital stay (ref). However, most studies on RM were underpowered to effectively assess clinical outcomes, either due to a low number of recruited patients or short duration of follow-up.

Recently, the PREDICT-RM trial demonstrated not only a reduction in all-cause rehospitalizations, but also in all-cause mortality in a large cohort of patients after newly ICD implantation who underwent RM during follow-up [6]. In the IN-TIME study, Hindricks, et al. [7] randomized 716 patients with left ventricular dysfunction submitted to implantation of an ICD (with or without CRT) to automatic, daily, implantbased telemonitoring versus conventional follow-up in a single-blinded design. After 1 year, patients under RM had a lower composite score of all-cause mortality, admission for HF, change in NYHA class, and patient global self-assessment. The secondary outcome of all-cause death was significantly lower in the RM group (10 vs. 27 patients).

The ALTITUDE Survival Study [8], a registry-based study which assessed the long-term survival of 194,006 ICD and CRT recipients of Boston intra-cardiac devices. Patients who were under RM follow-up with transmission of information to the LATITUDE network had significantly improved long-term survival, with a 50\% relative risk reduction on overall mortality (HR 0.56 for ICD recipients; HR 0.45 for CRT recipients). Also, the recently published EFFECT trial [9], a prospective, observational trial of RM in patients submitted to an ICD-CRT implantation, also showed an improvement in the combined endpoint of death or cardiovascular hospitalization.

Not all studies on the effect of RM on mortality have been positive. The TELE-HF trial [10] randomized 1653 high-risk patients to phone call telemonitoring or conventional follow-up. The main outcome of all-cause mortality or rehospitalization at 180 days was not significantly improved. However, the adherence and frequency of use of RM may have a significant impact on its clinical effect. A recently published systematic review and meta-analysis [11] of clinical outcomes in randomized controlled trials of RM vs. conventional follow up showed that a survival benefit was observed (OR: $0.65 ; \mathrm{p}=0.021$ ) only in the subset of 3 RCTs with daily verification of transmission, as is performed in our 


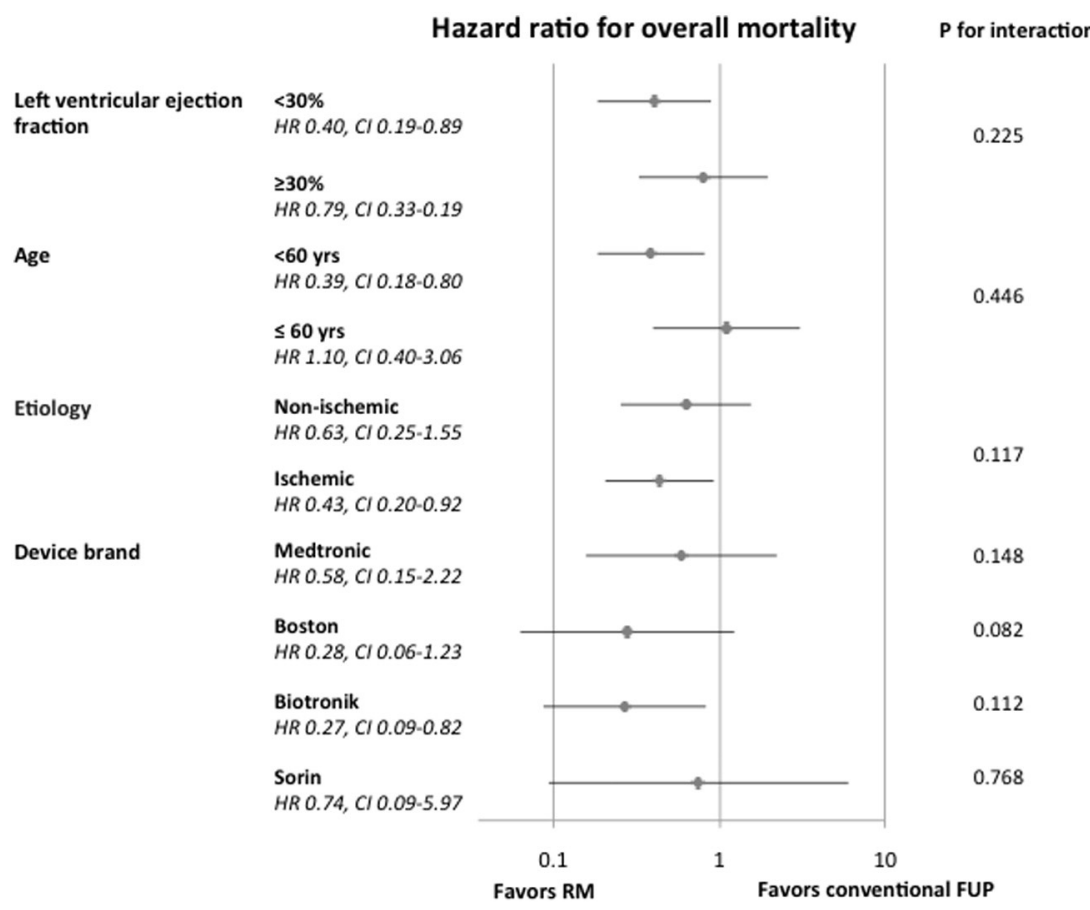

Fig. 2. Subgroup analysis for effect of RM on mortality.

center. In addition, a graded relationship between adherence to RM and improved survival was observed in a recent big data study of 269,471 implants by Varma, et al. [12].

In our study, patients with concomitant CRT were excluded. This population could conceivably have a similar or larger clinical benefit from RM, owning to the added complexity of their outpatient management. However, the effect of CRT on disease history and the variability in echocardiographic and clinical responses to CRT would make it more difficult to ascertain the exact effect of RM in these patients. In our study, patients who were subsequently upgraded to an ICD-CRT were censored from analysis after the procedure to exclude a potential confounding effect.

While the magnitude of the effect of RM on mortality in our study is surprising, it is similar to previous reports such as the IN-TIME study and the ALTITUDE survival registry. Suggested mechanisms for the mortality benefit observed with RM include earlier notification of adverse events, increased patient involvement in his own healthcare, and a

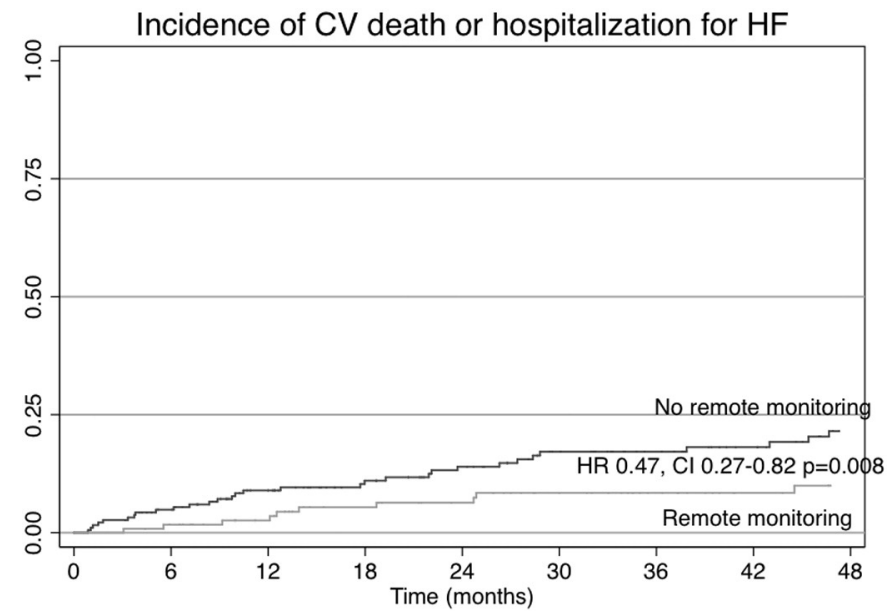

Fig. 3. Kaplan-Meier plot of incidence of cardiovascular death or hospitalization for heart failure. more comprehensive assessment of the cardiac health and arrhythmia status for each patient. In any case, our data supports the notion that RM is certainly not harmful, and may have a beneficial and long-term impact on overall mortality, which appears to stem mainly from lower cardiovascular mortality and a reduction in the incidence of hospitalizations for HF, a well recognized adverse prognostic factor.

\section{Limitations}

This was a single center non-randomized study, thus the generalizability of our results is limited. As this is a retrospective cohort trial, we cannot exclude the effect of unmeasured confounders or some degree of bias; in particular, despite there being no significant differences between both groups at baseline, we cannot exclude the effect of potential selection bias. In addition, since patients had to agree to the use of $\mathrm{RM}$, we could hypothesize that some of those who refused RM had also less adherence to other measures (i.e. medication), which could make RM a surrogate for non-compliance. However, measured patient characteristics were well balanced between groups at baseline, and the magnitude and temporal consistency of the clinical benefit on the RM group suggests a more significant effect that what would be explained by a lower compliance. In addition, the use of "hard" endpoints (hospitalization or death) and the employment of a publicly available database reduce the possibility of underreporting of events in the conventional follow-up group as compared to the RM group.

\section{Conclusions}

In this very long-term retrospective cohort study, the use of RM in patients submitted to implantation of an ICD for primary prevention was independently associated with increased survival. RM was also associated with a lower incidence of a composite endpoint of hospitalization for HF or cardiovascular mortality. Larger, adequately powered randomized clinical trials are warranted to definitely establish whether device-based remote monitoring can lead to improved outcomes in this high-risk population. 


\section{Conflict of interest}

The authors report no relationships that could be construed as a conflict of interest.

\section{References}

[1] N. Varma, A.E. Epstein, A. Irimpen, R. Schweikert, C. Love, Efficacy and safety of automatic remote monitoring for implantable cardioverter-defibrillator follow-up: the Lumos-T safely reduces routine office device follow-up (TRUST) trial, Circulation 122 (4) (Jul 27 2010) 325-332.

[2] A.M. Russo, R.F. Stainback, S.R. Bailey, A.E. Epstein, P.A. Heidenreich, M. Jessup, et al., ACCF/HRS/AHA/ASE/HFSA/SCAI/SCCT/SCMR 2013 appropriate use criteria for implantable cardioverter-defibrillators and cardiac resynchronization therapy: a report of the American College of Cardiology Foundation appropriate use criteria task force, Heart Rhythm. 10 (4) (Apr 2013) e11-e58.

[3] B.J. Gersh, B.J. Maron, R.O. Bonow, J.A. Dearani, M.A. Fifer, M.S. Link, et al., 2011 ACCF/AHA guideline for the diagnosis and treatment of hypertrophic cardiomyopathy: executive summary: a report of the American College of Cardiology Foundation/American Heart Association task force on practice guidelines, Circulation 124 (24) (Dec 13 2011) 2761-2796.

[4] N. Varma, J. Michalski, A.E. Epstein, R. Schweikert, Automatic remote monitoring of implantable cardioverter-defibrillator lead and generator performance: the Lumos-T safely RedUceS RouTine office device follow-up (TRUST) trial, Circ. Arrhythm. Electrophysiol. 3 (5) (Oct 2010) 428-436.
[5] P. Mabo, F. Victor, P. Bazin, S. Ahres, D. Babuty, A. Da Costa, et al., A randomized tria of long-term remote monitoring of pacemaker recipients (the COMPAS trial), Eur. Heart J. 33 (9) (May 29 2012) 1105-1111.

[6] J.G. Akar, H. Bao, P. Jones, Y. Wang, P. Varosy, F.A. Masoudi, et al., Use of remote monitoring is associated with lower risk of adverse outcomes among patients with implanted cardiac defibrillators, Circ. Arrhythm. Electrophysiol. (Jun 19 2015).

[7] G. Hindricks, M. Taborsky, M. Glikson, U. Heinrich, B. Schumacher, A. Katz, et al., Implant-based multiparameter telemonitoring of patients with heart failure (IN-TIME): a randomised controlled trial, Lancet 384 (9943) (Aug 16 2014) 583-590.

[8] L.A. Saxon, D.L. Hayes, F.R. Gilliam, P.A. Heidenreich, J. Day, M. Seth, et al., Long-term outcome after ICD and CRT implantation and influence of remote device follow-up: the ALTITUDE survival study, Circulation 122 (23) (Dec 7 2010) 2359-2367.

[9] A. De Simone, L. Leoni, M. Luzi, C. Amellone, G. Stabile, V. La Rocca, et al., Remote monitoring improves outcome after ICD implantation: the clinical efficacy in the management of heart failure (EFFECT) study, Europace 4 (Apr 2015).

[10] S.I. Chaudhry, J.A. Mattera, J.P. Curtis, J.A. Spertus, J. Herrin, Z. Lin, et al. Telemonitoring in patients with heart failure, N. Engl. J. Med. 363 (24) (Dec 9 2010) 2301-2309.

[11] N. Parthiban, A. Esterman, R. Mahajan, D.J. Twomey, R.K. Pathak, D.H. Lau, et al., Remote monitoring of implantable cardioverter-defibrillators: a systematic review and meta-analysis of clinical outcomes. J. Am. Coll. Cardiol. 65 (24) (May 8 2015) 2591-2600.

[12] N. Varma, J.P. Piccini, J. Snell, A. Fischer, N. Dalal, S. Mittal, Relationship between level of adherence to automatic wireless remote monitoring and survival in pacemaker and defibrillator patients, J. Am. Coll. Cardiol. 65 (24) (May 8 2015) 2601-2610. 